\title{
Fast Modified Vector Median Filter
}

\author{
B. Smolka ${ }^{1}$, M. Szczepanski ${ }^{1 \star}$, K.N. Plataniotis ${ }^{2}$, and A.N. Venetsanopoulos ${ }^{2}$ \\ 1 Silesian University of Technology \\ Department of Automatic Control \\ Akademicka 16 Str, 44-101 Gliwice, Poland \\ bsmolka@ia.polsl.gliwice.pl \\ 2 Edward S. Rogers Sr. Department of \\ Electrical and Computer Engineering \\ University of Toronto \\ 10 King's College Road, Toronto, Canada \\ kostas@dsp.toronto.edu
}

\begin{abstract}
A new filtering approach designed to eliminate impulsive noise in color images, while preserving fine image details is presented in this paper. The computational complexity of the new filter is significantly lower than that of the Vector Median Filter. The comparison shows that the new filter outperforms the VMF, as well as other standard procedures used in color image processing, when the impulse noise is to be eliminated.
\end{abstract}

Keywords: image processing, vector median filter

\section{Introduction}

The processing of color image data has received much attention in the last years. The amount of research published to date indicates an increasing interest in the area of color image processing [1-6]. It is widely accepted that color conveys very important information about the scene objects and this information can be used to further refine the performance of an imaging system.

The most common image processing tasks are noise filtering and image enhancement. These tasks are an essential part of any image processor whether the final image is utilized for visual interpretation or for automatic analysis [1, 2 .

As it has been generally recognized, that the nonlinear vector processing of color images is the most effective way to filter out noise and to enhance color images, the new filtering technique presented in the paper is also nonlinear and utilizes the correlation among the channels of a color image.

* This work was partially supported by KBN grant 8-T11E-013-19 
This paper is divided into three parts. In the first section, a brief overview of the standard noise reduction operations for color images based on the concept of vector median is presented. The second part shows the construction of the new algorithm of image enhancement and the last part depicts the results of noise attenuation achieved using the proposed algorithm in comparison with the standard noise suppression techniques described at the beginning of this paper.

\section{Standard Noise Reduction Filters}

A number of nonlinear, multichannel filters, which utilize correlation among multivariate vectors using various distance measures, have been proposed $[1,2]$. The most popular nonlinear, multichannel filters are based on the ordering of vectors in a predefined moving window. The output of these filters is defined as the lowest ranked vector according to a specific ordering technique.

Let $\mathbf{F}(x)$ represents a multichannel image and let $W$ be a window of finite size $n$ (filter length). The noisy image vectors inside the window $W$ are denoted as $\mathbf{F}_{j}, j=0,1, \ldots, n-1$. If the distance between two vectors $\mathbf{F}_{i}, \mathbf{F}_{j}$ is denoted as $\rho\left(\mathbf{F}_{i}, \mathbf{F}_{j}\right)$ then the scalar quantity

$$
R_{i}=\sum_{j=1}^{n} \rho\left(\mathbf{F}_{i}, \mathbf{F}_{j}\right),
$$

is the distance associated with the noisy vector $\mathbf{F}_{i}$ inside the processing window. An ordering of the $R_{i}$ 's

$$
R_{(0)} \leq R_{(1)} \leq \ldots \leq R_{(n-1)},
$$

implies the same ordering to the corresponding vectors $\mathbf{F}_{j}$

$$
\mathbf{F}_{(0)} \leq \mathbf{F}_{(1)} \leq \ldots \leq \mathbf{F}_{(n-1)} .
$$

Nonlinear ranked type multichannel estimators define the vector $\mathbf{F}_{(1)}$ as the filter output. This selection is due to the fact that vectors that diverge greatly from the data population usually appear in higher indexed locations in the ordered sequence. However, the concept of input ordering, initially applied in scalar quantities is not easily extended to multichannel data, since there is no universal way to define ordering in vector spaces.

To overcome the problem, distance functions are often utilized to order vectors. As an example, the Vector Median Filter (VMF) uses the $L_{1}$ norm in (1) to order vectors according to their relative magnitude differences [7].

The output of the VMF is the pixel $\mathbf{F}_{k} \in W$ for which the following condition is satisfied:

$$
\sum_{j=0}^{n-1} \rho\left(\mathbf{F}_{k}, \mathbf{F}_{j}\right)<\sum_{j=0}^{n-1} \rho\left(\mathbf{F}_{i}, \mathbf{F}_{j}\right), k \neq i .
$$


In this way the VMF consists of computing and comparing the values of $R_{i}$ and the output is the vector $\mathbf{F}_{k}$ for which $R$ reaches its minimum. In other words if for some $k$ the value

$$
R_{k}=\sum_{j=0}^{n-1} \rho\left(\mathbf{F}_{k}, \mathbf{F}_{j}\right)
$$

is smaller than $R_{0}$ :

$$
R_{0}=\sum_{j=0}^{n-1} \rho\left(\mathbf{F}_{0}, \mathbf{F}_{j}\right), \quad\left(\rho\left(\mathbf{F}_{k}, \mathbf{F}_{k}\right)=0\right)
$$

then the original value of the pixel $\mathbf{F}_{0}$ in the filter window $W$ is being replaced by $\mathbf{F}_{k}$ which satisfies the condition (4), which means that $k=\arg \min _{i} R_{i}$.

Figure $1 \mathrm{a}, \mathrm{b}$ ) shows the situation where the pixel $\mathbf{F}_{2}$ is moved to the center of the filter window and the original pixel $\mathbf{F}_{0}$ is replaced by $\mathbf{F}_{2}$ if the distance $R_{2}$ associated with $\mathbf{F}_{2}$ is smaller than $R_{0}$ and it is the minimal distance associated with the vectors belonging to the filtering window $W$.

The construction of the VMF is shown in Fig. 2, where the Euclidean distance is used. However different norms can be applied for noise suppression using the VMF concept. Most commonly the $L_{p}$ metric is used for the construction of the Vector Median Filter, then

$$
\rho\left(\mathbf{F}_{i}, \mathbf{F}_{j}\right)=\left\{\sum_{k=1}^{l}\left(F_{i}^{k}-F_{j}^{k}\right)^{p}\right\}^{\frac{1}{p}} .
$$

Choosing a specific value of $p$ we can obtain the following popular distance measures :

$$
\begin{gathered}
\rho\left(\mathbf{F}_{i}, \mathbf{F}_{j}\right)=\sum_{k=1}^{l}\left|F_{i}^{k}-F_{j}^{k}\right|, \quad p=1 \\
\rho\left(\mathbf{F}_{i}, \mathbf{F}_{j}\right)=\sqrt{\sum_{k=1}^{l}\left(F_{i}^{k}-F_{j}^{k}\right)^{2},} \quad p=2 \\
\rho\left(\mathbf{F}_{i}, \mathbf{F}_{j}\right)=\max _{k}\left|F_{i}^{k}-F_{j}^{k}\right|, \quad p=\infty
\end{gathered}
$$

where $l$ is the vector dimension. In this paper the $L_{1}, L_{2}$ and $L_{\infty}$ was used.

The Basic Vector Directional Filter (BVDF) is a ranked-order, nonlinear filter which parallelizes the VMF operation [8]. However, a distance criterion, different from the $L_{1}$ norm used in VMF, is utilized to rank the input vectors. The angular distance distance criterion used in BVDF is defined as a scalar measure

$$
R_{i}=\sum_{j=1}^{n} r\left(\mathbf{F}_{i}, \mathbf{F}_{j}\right), \quad \text { with } \quad r\left(\mathbf{F}_{i}, \mathbf{F}_{j}\right)=\cos ^{-1}\left(\frac{\mathbf{F}_{i} \mathbf{F}_{j}^{T}}{\left|\mathbf{F}_{i}\right|\left|\mathbf{F}_{j}\right|}\right)
$$




\begin{tabular}{|l|l|l|}
\hline & $\mathbf{F}_{1}$ & \\
\hline $\mathbf{F}_{4}$ & $\mathbf{F}_{0}$ & $\mathbf{F}_{2}$ \\
\hline & $\mathbf{F}_{3}$ & \\
\hline
\end{tabular}

a)

\begin{tabular}{|l|l|l|}
\hline & $\mathbf{F}_{1}$ & \\
\hline $\mathbf{F}_{4}$ & $\mathbf{F}_{2}$ & $\mathbf{F}_{0}$ \\
\hline & $\mathbf{F}_{3}$ & \\
\hline
\end{tabular}

b)

\begin{tabular}{|l|l|l|}
\hline & $F_{1}$ & \\
\hline$F_{4}$ & $F_{2}$ & $F_{2}$ \\
\hline & $F_{3}$ & \\
\hline
\end{tabular}

c)

Fig. 1. Illustration of the construction of the new filtering technique for the 4neighborhood system, as compared with the vector median. In case of the vector median the center pixel is replaced by its neighbor and the sum of distances between the center and neighbor pixel is being calculated a) and b). So the sum of distances is $R_{0}=\rho\left\{F_{0}, F_{1}\right\}+\rho\left\{F_{0}, F_{2}\right\}+\rho\left\{F_{0}, F_{3}\right\}+\rho\left\{F_{0}, F_{4}\right\}$ a) and $R_{2}=$ $\rho\left\{F_{2}, F_{1}\right\}+\rho\left\{F_{2}, F_{3}\right\}+\rho\left\{F_{2}, F_{4}\right\}+\rho\left\{F_{2}, F_{4}\right\}$ b). If $R_{2}<R_{0}$ then the temporary output of the vector median operator is $F_{2}$, then next pixels are being checked, whether they minimize the sum of distances. In the new method, if the center pixel $\mathbf{F}_{\mathbf{0}}$ is to be replaced by its neighbor $F_{2}$, then the pixel $\mathbf{F}_{\mathbf{0}}$ is removed from the filter window (c)) and the total distance $R_{2}=\rho\left\{F_{2}, F_{1}\right\}+\rho\left\{F_{2}, F_{3}\right\}+\rho\left\{F_{2}, F_{4}\right\}$ between $\mathbf{F}_{\mathbf{2}}$ (new center pixel) is calculated. If the total distance $R_{2}$ is greater than $R_{0}=-\beta+\rho\left\{F_{0}, F_{1}\right\}+\rho\left\{F_{0}, F_{2}\right\}+\rho\left\{F_{0}, F_{3}\right\}+\rho\left\{F_{0}, F_{4}\right\}$ then the center pixel is replaced by $\mathbf{F}_{2}$, otherwise it is retained.

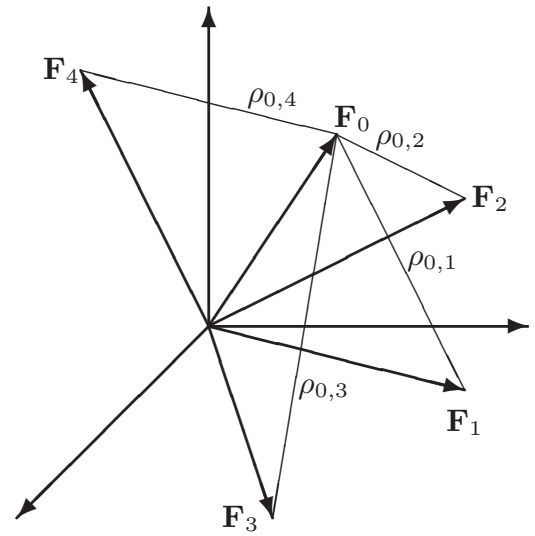

a)

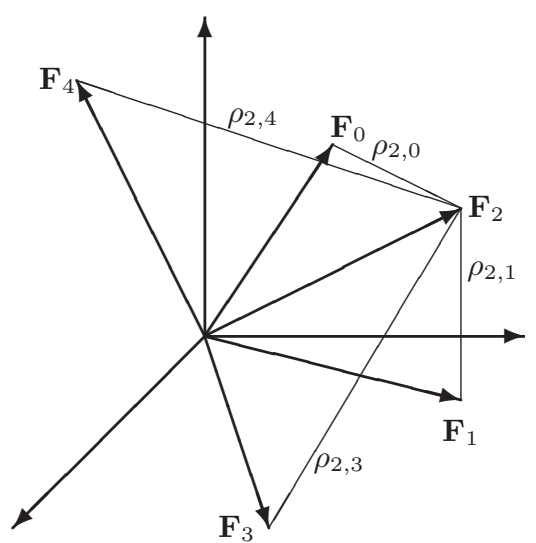

b)

Fig. 2. In the vector median filter, the distance $R_{0}$ associated with the vector $\mathbf{F}_{0}$ equals $R_{0}=\rho(0,1)+\rho(0,2)+\rho(0,3)+\rho(0,4)(\mathbf{a})$ and the distance $R_{2}$ associated with the vector $\mathbf{F}_{2}$ equals $R_{2}=\rho(2,0)+\rho(2,1)+\rho(2,3)+\rho(2,4)$ (b). 
as the distance associated with the noisy vector $\mathbf{F}_{i}$ inside the processing window of length $n$. The output of the BVDF is that vector from the input set, which minimizes the sum of the angles with the other vectors. In other words, the BVDF chooses the vector most centrally located without considering the magnitudes of the input vectors.

To improve the efficiency of the directional filters, a new method called Directional-Distance Filter (DDF) was proposed [10]. This filter retains the structure of the BVDF but utilizes a new distance criterion to order the vectors inside the processing window.

The new rank-ordered operation called Hybrid Directional Filter was proposed in 11]. This filter operates on the directional and the magnitude of the color vectors independently and then combines them to produce a unique final output. This hybrid filter, which can be viewed as a nonlinear combination of the VMF and BVDF filters, produces an output according to the following rule:

$$
\mathbf{F}_{H y F}= \begin{cases}\mathbf{F}_{V M F} & \text { if } \mathbf{F}_{V M F}=\mathbf{F}_{B V D F} \\ \left(\frac{\left\|\mathbf{F}_{V M F}\right\|}{\left\|\mathbf{F}_{B V D F}\right\|}\right) \mathbf{F}_{B V D F} & \text { otherwise }\end{cases}
$$

where $\mathbf{F}_{B V D F}$ is the output of the BVDF filter, $\mathbf{F}_{V M F}$ is the output of the $\mathrm{VMF}$ and $\|\cdot\|$ denotes the magnitude of the vector.

A different approach in the development of directional filters was taken with the introduction of a new class of adaptive directional filters [1415]. The adaptive filters proposed there, utilize data-dependent coefficients to adapt to local image characteristics. The weights of the adaptive filters are determined by fuzzy transformations based on features from local data.

The general form of the adaptive directional filters is given as a nonlinear transformation of a weighted average of input vectors inside the filter window. In the adaptive design, the weights $w_{i}$ provide the degree to which an input vector contributes to the output of the filter. The weights of the filter are determined adaptively using fuzzy transformations of a distance criterion at each image position.

\section{Fast Modified Vector Median Filter (FMVMF)}

The construction of the new filter (FMVMF) is very similar to that of the VMF. Let the distance associated with the center pixel be

$$
R_{0}=-\beta+\sum_{j=1}^{n-1} \rho\left(\mathbf{F}_{0}, \mathbf{F}_{j}\right)
$$

where $\beta$ is a threshold parameter and let the distance associated with the neighbors of $\mathbf{F}_{0}$ be

$$
R_{i}=\sum_{j=1}^{n-1} \rho\left(\mathbf{F}_{i}, \mathbf{F}_{j}\right), i=1, \ldots, n-1
$$


Then, if for some $k, R_{k}$ is smaller than $R_{0}$

$$
R_{k}=\sum_{j=1}^{n-1} \rho\left(\mathbf{F}_{k}, \mathbf{F}_{j}\right)<R_{0},
$$

then $\mathbf{F}_{0}$ is being replaced by $\mathbf{F}_{k}$. It happens when

$$
\sum_{j=1}^{n-1} \rho\left(\mathbf{F}_{k}, \mathbf{F}_{j}\right)<-\beta+\sum_{j=1}^{n-1} \rho\left(\mathbf{F}_{0}, \mathbf{F}_{j}\right),
$$

so the condition is

$$
\beta<\sum_{j=1}^{n-1}\left\{\rho\left(\mathbf{F}_{0}, \mathbf{F}_{j}\right)-\rho\left(\mathbf{F}_{k}, \mathbf{F}_{j}\right)\right\} .
$$

The construction of the new filter is very similar to that of VMF, however the major difference is presented in 1c) and Fig. 5. The pixel $\mathbf{F}_{2}$ is put to the center of $W$ and the center pixel $\mathbf{F}_{0}$ is removed from the window. The center pixel $\mathbf{F}_{0}$ will be replaced by $\mathbf{F}_{2}$ if the distance $R_{2}$ associated with $\mathbf{F}_{2}$ is smaller than $R_{0}$ and is the minimal distance associated with the vectors belonging to $W$.

The rejection of the center pixel $\mathbf{F}_{0}$ is the most important feature of the new algorithm. As the center pixel is suspected to be noisy, it is not taken into account when calculating the distances associated with the neighbors of $\mathbf{F}_{0}$. This is illustrated in Fig. 1 c) and Fig. 5.

Table 1. Filters taken for comparison with the new modified Vector median filter.

\begin{tabular}{|c|c|c|}
\hline Notation & Filter & Ref. \\
\hline AMF & Arithmetic Mean & {$[2]$} \\
\hline VMF & Vector Median & {$[7]$} \\
\hline BVDF & Basic Vector Directional & {$[8]$} \\
\hline GVDF & $\begin{array}{c}\text { Generalized Vector } \\
\text { Directional }\end{array}$ & {$[8]$} \\
\hline DDF & Directional-Distance & {$[5]$} \\
\hline HDF & Hybrid Directional & {$[9]$} \\
\hline AHDF & Adapt. Hybrid Directional & {$[9]$} \\
\hline FVDF & Fuzzy Vector Directional & {$[10]$} \\
\hline ANNF & Adapt. Nearest Neighbor & {$[11]$} \\
\hline ANP-EF & $\begin{array}{c}\text { Adapt. Non-Param. } \\
\text { (Exponential) }\end{array}$ & {$[14]$} \\
\hline ANP-GF & $\begin{array}{c}\text { Adapt. Non-Param. } \\
\text { (Gaussian) }\end{array}$ & {$[14]$} \\
\hline ANP-DF & $\begin{array}{c}\text { Adapt. Non-Param. } \\
\text { (Directional) }\end{array}$ & {$[14]$} \\
\hline
\end{tabular}

In finding the maximum in (5), we obtain $n-1$ nonzero components in $R_{0}$. If we replace the central pixel by one of its neighborhood (by $\mathbf{F}_{2}$ in Figs. 1 and 4), then we obtain only $n-2$ nonzero components in $R$ (14), as the center pixel disappears from the filter window. In this way the filter is faster than the VMF and replaces the central pixel only when it is really noisy while preserving the original undisturbed image structures. 

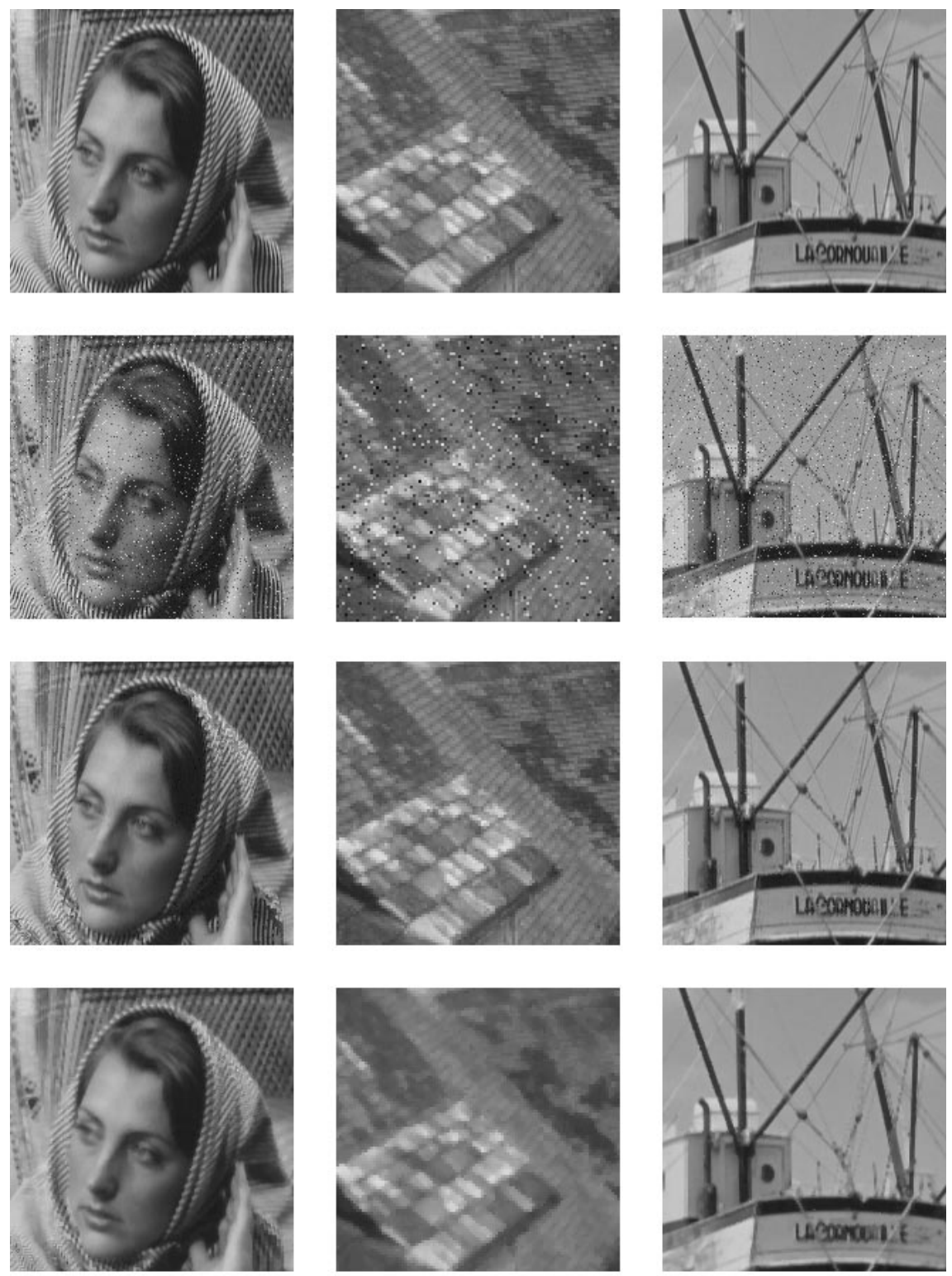

Fig. 3. Comparison of the efficiency of the vector median and the proposed filter: a) parts of the BARBARA, GOLDHIL and BOATS color images, b) images contaminated by $4 \%$ impulsive noise, c) images filtered using the proposed technique, d) the result of the filtering with the vector median. 


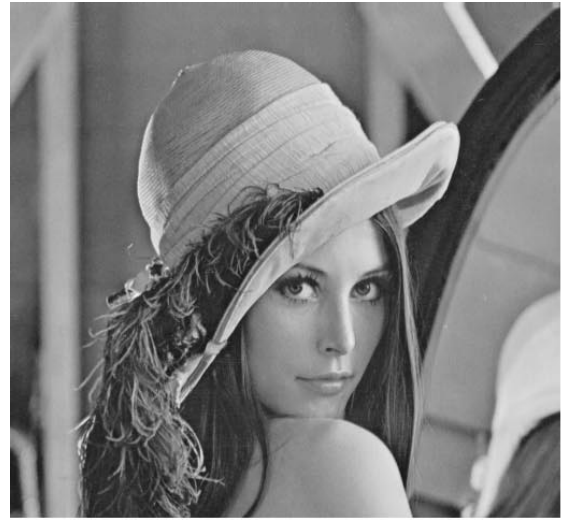

a)

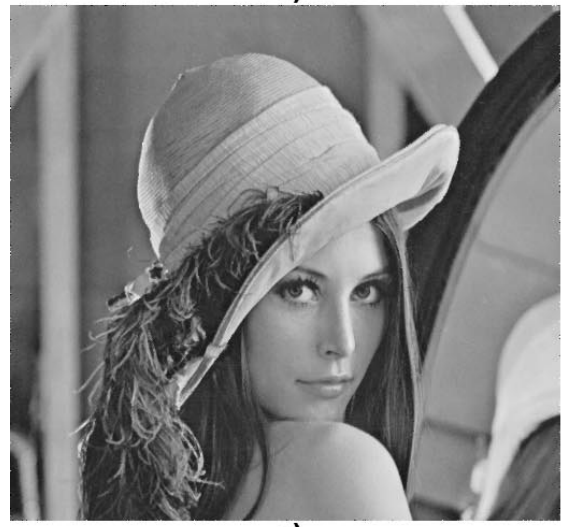

c)

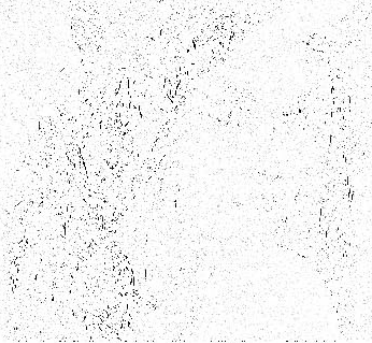

e)

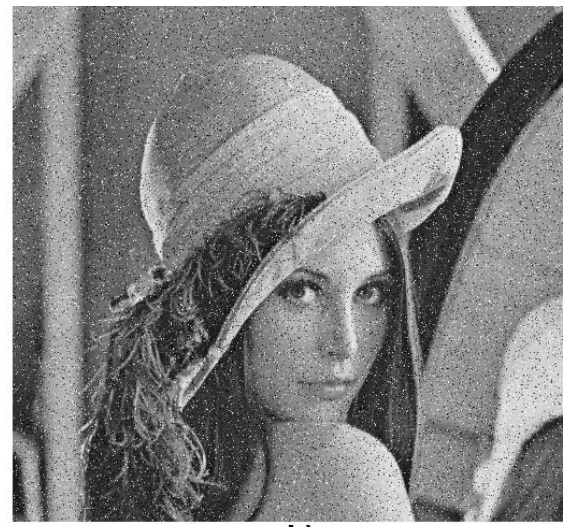

b)

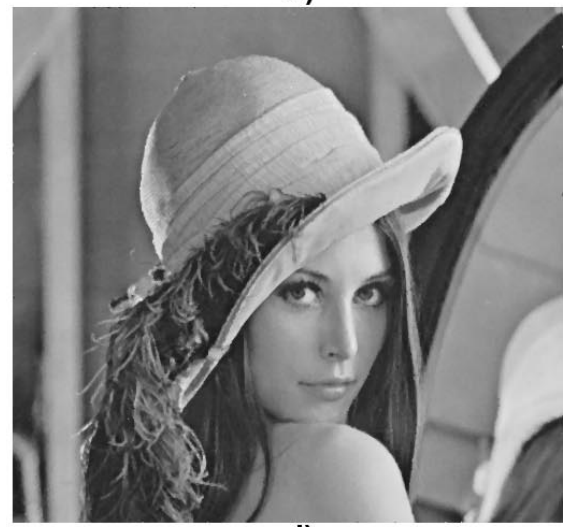

d)

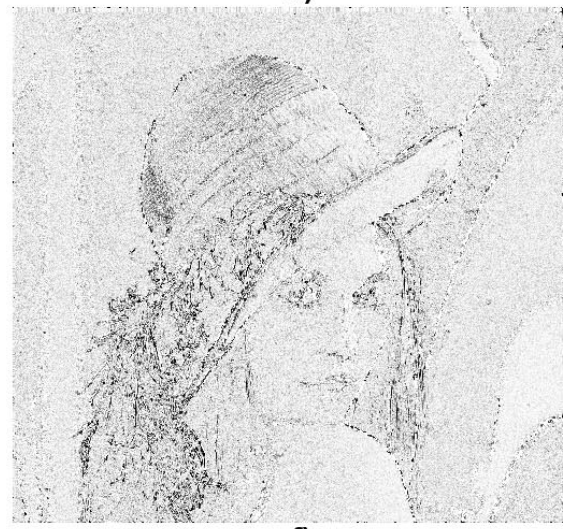

f)

Fig. 4. Noise reduction effect of the proposed filter as compared with the standard VMF: a) colour test image $L E N A$, b) image distorted by $4 \%$ impulsive noise, c) new method $(3 \times 3$ window, $\beta=0.5)$, d) VMF, e) and f) the absolute difference between the original and filtered image (the RGB values were multiplied by factor 10 ). 
Table 2. Comparison of the new algorithm $\left(\rho_{0}=0.75\right)$ with the standard techniques (Tab. 1) using the LENA standard image.

\begin{tabular}{|c|c|c|c|c|c|}
\hline $\begin{array}{c}\text { FILTER } \\
\text { LENA }\end{array}$ & $\begin{array}{c}\text { NMSE } \\
{\left[10^{-3}\right]}\end{array}$ & $\mathbf{R M S E}$ & $\begin{array}{c}\text { SNR } \\
{[\mathbf{d B}]}\end{array}$ & $\begin{array}{c}\text { PSNR } \\
{[\mathbf{d B}]}\end{array}$ & $\begin{array}{c}\text { NCD } \\
{\left[10^{-4}\right]}\end{array}$ \\
\hline NONE & 514.72 & 32.17 & 12.88 & 17.98 & 79.17 \\
\hline AMF & 79.32 & 12.63 & 21.01 & 26.11 & 82.75 \\
\hline VMF & 18.77 & 6.14 & 27.27 & 32.375 & 40.47 \\
\hline BVDF & 24.59 & 7.03 & 26.09 & 31.19 & 41.15 \\
\hline GVDF & 19.47 & 6.26 & 27.11 & 32.20 & 41.77 \\
\hline DDF & 18.87 & 6.16 & 27.24 & 32.34 & 40.24 \\
\hline HDF & 18.61 & 6.12 & 27.30 & 32.40 & 41.28 \\
\hline AHDF & 18.31 & 6.07 & 27.37 & 32.47 & 41.17 \\
\hline FVDF & 22.25 & 6.69 & 26.53 & 31.63 & 44.69 \\
\hline ANNF & 26.80 & 7.34 & 25.72 & 30.82 & 48.01 \\
\hline ANP-E & 78.60 & 12.57 & 21.05 & 26.14 & 82.46 \\
\hline ANP-G & 78.62 & 12.57 & 21.05 & 26.14 & 82.48 \\
\hline ANP-D & 24.18 & 6.97 & 26.17 & 31.26 & 46.07 \\
\hline NEW- $L_{1}$ & $\mathbf{4 . 9 9}$ & $\mathbf{3 . 1 7}$ & $\mathbf{3 3 . 0 1}$ & $\mathbf{3 8 . 1 1}$ & $\mathbf{6 . 6 2}$ \\
\hline NEW- $L_{2}$ & $\mathbf{5 . 2 4}$ & $\mathbf{3 . 2 5 6}$ & $\mathbf{3 2 . 8 0}$ & $\mathbf{3 7 . 9 0}$ & $\mathbf{6 . 5 1}$ \\
\hline NEW- $L_{\infty}$ & $\mathbf{5 . 2 4}$ & $\mathbf{3 . 2 5}$ & $\mathbf{3 2 . 8 0}$ & $\mathbf{3 7 . 9 0}$ & $\mathbf{6 . 5 1}$ \\
\hline
\end{tabular}

\section{Results}

The color images Lena and Peppers have been contaminated by $4 \%$ impulsive noise. The root of the mean squared error (RMSE), signal to noise ratio (SNR), peak signal to noise ratio (PSNR), normalized mean square error (NMSE) and normalized color difference (NCD) [3] were analyzed. The comparison shows that the new filter outperforms by far the standard vector median filter (which can be treated as a reference filter), when the impulsive noise has to be eliminated. The efficiency of the new filtering technique is shown in Tabs. 2 and 3 and in Figs. 3 and 4.

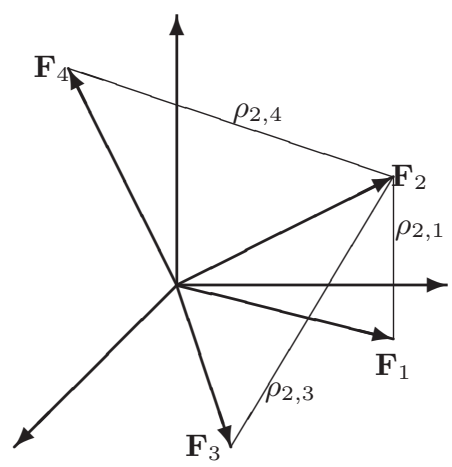

Fig. 5. In the new method the distance $R_{2}$ equals $R_{2}=\rho(2,1)+\rho(2,3)+\rho(2,4)$. 
The threshold $\beta$ in (13) is a design parameter of the new filter. For $\beta=0$ similar results to that obtained with the standard VMF have been achieved. However if $\beta>0$ then the new filter has the ability of noise removal while preserving fine image details (lines, edges, corners, texture). If the RGB values of the color image are normalized to the range $[0,1]$, then good filtering results have been achieved for $\beta$ from the $[0.5,0.75]$ interval.

Table 3. Comparison of the new algorithm $\left(\rho_{0}=0.75\right)$ with the standard techniques (Tab. 1) using the PEPPERS standard image.

\begin{tabular}{|c|c|c|c|c|c|}
\hline $\begin{array}{c}\text { FILTER } \\
\text { PEPPER } S\end{array}$ & $\begin{array}{c}\text { NMSE } \\
{\left[10^{-3}\right]}\end{array}$ & $\mathbf{R M S E}$ & $\begin{array}{c}\text { SNR } \\
{[\mathbf{d B}]}\end{array}$ & $\begin{array}{c}\text { PSNR } \\
{[\mathbf{d B}]}\end{array}$ & $\begin{array}{c}\text { NCD } \\
{\left[10^{-4}\right]}\end{array}$ \\
\hline NONE: & 650.33 & 32.63 & 11.87 & 17.86 & 75.23 \\
\hline AMF & 108.65 & 13.34 & 19.64 & 25.63 & 100.47 \\
\hline VMF & 27.57 & 6.72 & 25.60 & 31.59 & 51.64 \\
\hline BVDF & 47.94 & 8.86 & 23.19 & 29.18 & 54.67 \\
\hline GVDF & 31.86 & 7.22 & 24.97 & 30.96 & 52.70 \\
\hline DDF & 28.18 & 6.79 & 25.50 & 31.49 & 51.14 \\
\hline HDF & 26.82 & 6.63 & 25.72 & 31.71 & 51.42 \\
\hline AHDF & 26.43 & 6.58 & 25.78 & 31.77 & 51.32 \\
\hline FVDF & 33.34 & 7.39 & 24.77 & 30.76 & 54.07 \\
\hline ANNF & 45.12 & 8.59 & 23.46 & 29.45 & 65.89 \\
\hline ANP-DF & 37.24 & 7.81 & 24.29 & 30.28 & 56.39 \\
\hline ANP-EF & 106.70 & 13.22 & 19.72 & 25.71 & 99.76 \\
\hline ANP-GF & 106.69 & 13.22 & 19.72 & 25.71 & 99.75 \\
\hline NEW- $L_{1}$ & $\mathbf{8 . 9 3}$ & $\mathbf{3 . 8 2}$ & $\mathbf{3 0 . 4 9}$ & $\mathbf{3 6 . 4 8}$ & $\mathbf{9 . 8 1}$ \\
\hline NEW- $L_{2}$ & $\mathbf{8 . 5 1}$ & $\mathbf{3 . 7 3}$ & $\mathbf{3 0 . 7 0}$ & $\mathbf{3 6 . 6 9}$ & $\mathbf{8 . 7 1}$ \\
\hline NEW- $L_{\infty}$ & $\mathbf{8 . 5 1}$ & $\mathbf{3 . 7 3}$ & $\mathbf{3 0 . 7 0}$ & $\mathbf{3 6 . 6 9}$ & $\mathbf{8 . 7 1}$ \\
\hline
\end{tabular}

\section{Conclusions}

The new algorithm presented in this paper can be seen as a modification and improvement of the commonly used vector median filter. The computational complexity of the new filter is significantly lower than that of the Vector Median. The comparison shows that the new filter outperforms the VMF, as well as other standard procedures used in color image processing, when the impulse noise should be eliminated.

\section{References}

1. Pitas, I., Venetsanopoulos, A. N.: Nonlinear Digital Filters: Principles and Applications. Kluwer Academic Publishers, Boston, MA (1990)

2. Plataniotis, K.N., Venetsanopoulos, A.N.: Color Image Processing and Applications. Springer Verlag (June 2000)

3. Venetsanopoulos, A.N., Plataniotis, K.N.: Multichannel image processing. Proceedings of the IEEE Workshop on Nonlinear Signal/Image Processing 2-6 (1995)

4. Pitas, I., Tsakalides, P.: Multivariate ordering in color image processing. IEEE Trans. on Circuits and Systems for Video Technology 1, 3 (1991) 247-256 
5. Karakos, D.G., Trahanias, P.E.: Combining vector median and vector directional filters: The directional-distance filters. Proceedings of the IEEE Conf. on Image Processing, ICIP-95 (Oct 1995) 171-174

6. Pitas, I., Venetsanopoulos, A.N.: Order statistics in digital image processing. Proceedings of IEEE 80(12) (1992) 1893-1923

7. Astola, J., Haavisto, P., Neuovo, Y.: Vector median filters. IEEE Proceedings 78 (1990) 678-689

8. Trahanias, P.E., Venetsanopoulos, A.N.: Vector directional filters: A new class of multichannel image processing filters. IEEE Trans. on Image Processing 2(4) (1993) 528-534

9. Trahanias, P.E., Karakos, D., Venetsanopoulos, A.N.: Directional processing of color images : theory and experimental results. IEEE Trans. on Image Processing 5(6) (1996) 868-880

10. Karakos, D., Trahanias, P.E.: Generalized multichannel image filtering structures. IEEE Trans. on Image Processing 6(7) (1997) 1038-1045

11. Gabbouj, M., Cheickh, F.A.: Vector median - vector directional hybrid filter for colour image restoration. Proceedings of EUSIPCO (1996) 879-881

12. Plataniotis, K. N., Androutsos, D., Venetsanopoulos, A.N.V.: Fuzzy adaptive filters for multichannel image processing. Signal Processing Journal 55(1) (1996) 93-106

13. Plataniotis, K. N., Androutsos, D., Sri, V., Venetsanopoulos, A.N.V.: A nearest neighbour multichannel filter. Electronic Letters (1995) 1910-1911

14. Plataniotis, K.N., Androutsos, D., Vinayagamoorthy, S., Venetsanopoulos, A.N.V.: Color image processing using adaptive multichannel filters. IEEE Trans. on Image Processing 6(7) (1997) 933-950

15. Plataniotis, K.N., Androutsos, D., Venetsanopoulos, A.N.V.: Colour Image Processing Using Fuzzy Vector Directional Filters. Proc. of the IEEE Workshop on Nonlinear Signal/Image Processing. Greece (1995) 535-538 